\title{
About the torque and energy balances for the flow between eccentric rotating disks
}

\author{
R. J. J. Jongschaap and P. F. Mijnlieff
}

With 1 figure

(Received October 21, 1976)

\section{Notation}

a relative horizontal displacement of the centers of the disks

$A$ rate of work delivered to the fluid surfaces (eqs. [19]-[21])

$D \quad$ rate of strain tensor (eq. [4])

$h$ distance between the plates

$M \quad$ torque exerted on the fluid (eq. [12])

$n \quad$ local unit normal on the fluid surface

r $\quad$ radius vector (eq. [13])

$r, \theta, z \quad$ skew cylindrical coordinates (eq. [1])

$R \quad$ radius of the disks

$S \quad$ vertical component of the torque exerted on a horizontal circle in the free surface (used in eq. [A 2])

$t \quad$ local traction on the fluid surface (eqs. [9]-[11])

$T$ stress tensor

$v \quad$ local fluid velocity (eq. [3])

$w \quad$ rate of energy dissipation per unit volume (eq. [17])

$W \quad$ total rate of energy dissipation in the fluid (eq. [18])

$x, y, z$ Cartesian coordinates

$\eta^{\prime} \quad$ real part of the complex viscosity

$\eta^{\prime \prime} \quad$ negative imaginary part of the complex viscosity

$\psi \quad$ dimensionless measure of eccentricity (eq. [2])

$\omega$ angular velocity of the rotation of the disks

Special symbols

$\boldsymbol{A}^{T} \quad$ transpose of a tensor $\boldsymbol{A}$

$A\langle i j\rangle$ Cartesian components of a tensor $A$

$v\langle i\rangle$ Cartesian components of a vector $v$

\section{Subscripts}

$\begin{array}{ll}f & \text { free surface } \\ l & \text { lower surface } \\ u & \text { upper surface }\end{array}$

\section{Introduction}

Although the eccentric rotating disks (ERD) or Maxwell orthogonal rheometer has already become a familiar flow configuration, some fundamental questions about the torque and energy balances seem to have been not yet fully clarified.
First there is a point discussed by Payvar and Tanner (1) and by Davis and Macosco (2) They pointed out that if the flow field is as proposed first by Blyer and Kurtz (3) the total torque acting on the sample differs from zero. This made them assume that a torsional flow was superimposed on the primarily assumed flow, in such a way that the torque balance was restored.

A second point is the so-called 'stress power paradox' (4). By this is meant the observation that, although energy is dissipated in the sample, no power seems to enter into the material from the apparatus. Again one arrives at the conclusion that the actual flow field should differ from the primarily assumed one.

In this paper both points will be discussed again. Special attention will be given to the free surface. Briefly, this has the following reason. The medium surrounding the instrument can only exert an isotropic pressure on the free surface, whatever its shape is. The stresses in the material at the free surface, however, are not isotropic, at least not if the velocity field near the free surface is still exactly the same as the one assumed in the bulk. Discontinuities in stress are unacceptable, however. It will be shown that neither deviations from the assumed shape (that of a skew cylinder) of the free surface, nor the superposition of a torsional flow, nor a combination of these two mechanisms are able to remove the apparent discontinuity. So a third mechanism must be thought of. As such we will propose that the velocity field in a layer along the free surface differs from that in the bulk. In this layer, due to the presence of a non-homogeneous flow field, the stress changes from an isotropic one at the free surface to the non-isotropic stress that prevails in the bulk. 
If the free surface layer in which this happens is relatively thin, the stress it delivers to the bulk may, formally, be treated as being exerted on the free surface itself, by a two-dimensional skin.

Prior to discussing how the actual flow field might be we will show that if the stresses at the free surface are taken into account no inconsistencies in the torque and energy balances exist.

\section{Preliminaries}

The essential features of the ERD-flow are shown in fig. 1. A Cartesian coordinate frame (fixed in space) is located with its origin in the center of the lower disk and its $z$-axis perpen-

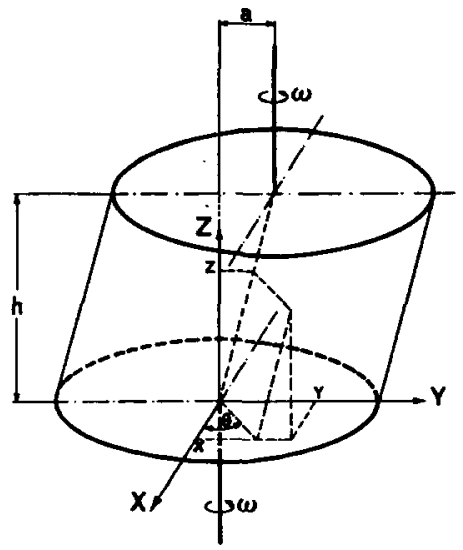

Fig. 1. Geometry of the ERD-flow.

dicular to it. The upper disk is shifted with respect to the lower one by an amount $a$ in the $y$-direction; the distance $h$ between the plates is kept constant. The upper disk is driven at a constant angular velocity $\omega$, the lower disk is assumed to follow at the same speed. Besides the Cartesian coordinates we also use coordinates $r$, $\theta$ and $z$, defined by:

$x=r \cos \theta$,

$y=r \sin \theta+\psi z$,

$z=z$

where

$\psi=\frac{a}{h}$.

In this paper we only consider the case that inertia effects can be neglected; therefore Cartesian components of the velocity field are assumed to be given by (3): $\boldsymbol{v}=(-\omega y+\omega \psi z, \omega x, 0)$.

A discussion of the influence of inertia effects on the velocity fields has been given by $A b b o t t$ and Walters (7) and recently also by Waterman (8).

The Cartesian components of the rate of strain tensor $D$, defined as

$D=\frac{1}{2}\left(\operatorname{grad} v+(\operatorname{grad} v)^{T}\right)$,

become:

$[D\langle i j\rangle]=\left[\begin{array}{lll}0 & 0 & \frac{1}{2} \omega \psi \\ 0 & 0 & 0 \\ \frac{1}{2} \omega \psi & 0 & 0\end{array}\right]$.

The shape of the free surface is assumed (5) to be that of a skew cylinder with radius $R$ around the axis $r=0$; its Cartesian coordinates are given by:

$x=R \cos \theta$

$y=R \sin \theta+\psi z$

$0 \leq \theta \leq 2 \pi, 0 \leq z \leq h$.

The Cartesian components of the unit normal $\boldsymbol{n}_{\boldsymbol{f}}$ on this surface are:

$$
\begin{aligned}
\boldsymbol{n}_{f}= & {\left[\frac{\cos \theta}{\sqrt{1+\psi^{2} \sin ^{2} \theta}}, \frac{\sin \theta}{\sqrt{1+\psi^{2} \sin ^{2} \theta}},\right.} \\
& \left.-\frac{\psi \sin \theta}{\sqrt{1+\psi^{2} \sin ^{2} \theta}}\right] .
\end{aligned}
$$

Since inertia effects are neglected the pressure term in the stress response will be chosen to be equal to zero. In the region of linear viscoelastic response the stress tensor then becomes (6):

$[T\langle i j\rangle]=\left[\begin{array}{lll}0 & 0 & \omega \psi \eta^{\prime} \\ 0 & 0 & \omega \psi \eta^{\prime \prime} \\ \omega \psi \eta^{\prime} & \omega \psi \eta^{\prime \prime} & 0\end{array}\right]$

in which $\eta^{\prime}$ and $\eta^{\prime \prime}$ are, respectively, the real and the negative imaginary part of the complex viscosity.

From [8] the stresses $t_{u}$ and $t_{l}$ on the upper and the lower surface of the fluid are seen to be

$\boldsymbol{t}_{u}=\boldsymbol{T} \cdot \boldsymbol{n}_{u}=\left(\omega \psi \eta^{\prime}, \omega \psi \eta^{\prime \prime}, 0\right)$,

$\boldsymbol{t}_{l}=\boldsymbol{T} \cdot \boldsymbol{n}_{l}=\left(-\omega \psi \eta^{\prime},-\omega \psi \eta^{\prime \prime}, 0\right)$,

respectively, whereas - by [7] and [8] - the stress on the free surface becomes: 


$$
\begin{aligned}
& \boldsymbol{t}_{f}=\boldsymbol{T} \cdot \boldsymbol{n}_{f}=\left[\frac{-\omega \psi^{2} \eta^{\prime} \sin \theta}{\sqrt{1+\psi^{2} \sin ^{2} \theta}}, \frac{-\omega \psi^{2} \eta^{\prime \prime} \sin \theta}{\sqrt{1+\psi^{2} \sin ^{2} \theta}},\right. \\
& \left.\frac{\omega \psi\left(\eta^{\prime} \cos \theta+\eta^{\prime \prime} \sin \theta\right)}{\sqrt{1+\psi^{2} \sin ^{2} \theta}}\right] \text {. }
\end{aligned}
$$

Since no forces are delivered to the free surface and a stress given by [11] is required, there is a discontinuity of stress at that surface. So, as it has been mentioned in the Introduction, there should be a mechanism which removes this discontinuity. For the moment we will bypass the questions about the nature of such a mechanism, and investigate the case that the stress given by [11] is indeed exerted on the free surface; the shape of this surface is supposed to be given by eq. [6].

\section{Torque balance}

The torque with respect to the center of the lower disk on any of the surfaces $S$ of the fluid may be calculated from

$$
\boldsymbol{M}=\int_{S} \boldsymbol{r} \times \boldsymbol{t} d S,
$$

in which $\boldsymbol{r}$ is the radius vector pointing from the origin to a point of the surface:

$\boldsymbol{r}=(x, y, z)=(r \cos \theta, r \sin \theta+\psi z, z)$.

With [12] the $z$-component of the torque on the upper surface of the fluid is calculated to be

$$
\begin{aligned}
M_{u}\langle z\rangle= & \int_{0}^{R} \int_{0}^{2 \pi}\left\{\omega \psi \eta^{\prime \prime} r \cos \theta-\omega \psi \eta^{\prime}\right. \\
& \cdot(r \sin \theta+\psi h) r d r d \theta \\
= & -\pi R^{2} \psi^{2} h \omega \eta^{\prime} ;
\end{aligned}
$$

on the lower surface:

$$
\begin{aligned}
M_{l}\langle z\rangle= & \int_{0}^{R} \int_{0}^{2 \pi}\left\{\omega \psi \eta^{\prime \prime} r \cos \theta-\omega \psi \eta^{\prime} r \sin \theta\right\} \\
& \cdot r d r d \theta=0 ;
\end{aligned}
$$

and on the free surface:

$$
\begin{aligned}
M_{f}\langle z\rangle= & \int_{0}^{2 \pi} \int_{0}^{R}\left\{-\omega \psi^{2} \eta^{\prime \prime} \sin \theta R \cos \theta\right. \\
& \left.+\omega \psi \eta^{\prime} \sin \theta(R \sin \theta+\psi z)\right\} R d \theta d z \\
= & \pi R^{2} \psi^{2} h \omega \eta^{\prime} .
\end{aligned}
$$

Here [11] has been used, in combination with the fact that an infinitesimal element of the free surface is given by $\sqrt{1+\psi^{2} \sin ^{2} \theta} R d \theta d z$.
We see that when only the tractions on the disks are considered the torque [14] is not compensated. It was for this reason that Payvar and Tanner (1), and Davis and Macosco (2), assumed that a torsional flow, leading to an additional torque on the upper disk equal and opposite to $M_{u}\langle z\rangle$ given by eq. [14], was superimposed on the velocity field [3]. From [16], however, it is clear that in the case considered here the required compensation is taken care of by the stresses on the free surface.

\section{Energy balance}

The rate of energy dissipation in the ERDflow is calculated from [5] and [8] to be per unit volume

$w=\sum_{i, j} T\langle i j\rangle D\langle i j\rangle=\omega^{2} \psi^{2} \eta^{\prime}$

and for the total amount of fluid:

$W=\pi R^{2} h \omega^{2} \psi^{2} \eta^{\prime}$.

On the other hand by [3] and [9] the rate of work done on the upper surface of the fluid becomes

$$
\begin{aligned}
A_{u}= & \int_{S_{u}} \boldsymbol{v} \cdot \boldsymbol{t}_{u} d S_{u} \\
= & \int_{S_{u}}\left\{(-\omega y+\omega \psi h) \omega \psi \eta^{\prime}+\omega^{2} \psi x \eta^{\prime \prime}\right\} d S_{u} \\
= & \int_{0}^{R} \int_{0}^{2 \pi}\left(-\omega^{2} \psi \eta^{\prime} r \sin \theta\right. \\
& \left.+\omega^{2} \psi \eta^{\prime \prime} r \cos \theta\right) r d r d \theta=0 .
\end{aligned}
$$

In the same way the rate of work delivered to the lower surface of the fluid is found to be

$A_{l}=\int_{S_{l}} v \cdot t_{l}=0$.

This seems to bring us the paradoxical result that energy is dissipated in the material although no power seems to enter into it from the apparatus. We still have to calculate, however, the contribution $A_{f}$ of the free surface to the rate of work done on the material. Using [3] and $[11]$ this is calculated to be

$$
\begin{aligned}
A_{f}= & \int_{S_{f}} \boldsymbol{v} \cdot \boldsymbol{t}_{f} d S_{f} \\
= & \int_{0}^{d} \int_{0}^{2 \pi}\{(-\omega(R \sin \theta+\psi z)+\omega \psi z) \\
& \cdot\left(-\omega \psi^{2} \eta^{\prime} \sin \theta\right)+(\omega R \cos \theta) \\
& \left.\cdot\left(-\omega \psi^{2} \eta^{\prime \prime} \sin \theta\right)\right\} R d \theta d z \\
= & \pi R^{2} h \omega^{2} \psi^{2} \eta^{\prime},
\end{aligned}
$$


which indeed equals the total rate of energy dissipation [18].

\section{Discussion}

In the preceding sections it has been shown that the balances of torque and energy are satisfied, provided the flow field is as given by [3] and the free surface has a shape described by [6]. The role of the free surface appeared to be essential: the stresses at this surface were able to compensate the torque exerted on the sample by the upper plate. Furthermore all the energy dissipated in the sample appeared to have entered as work done on it by stresses on the free surface.

So the assumptions used do not lead to internal contradictions. This, however, does not mean that those assumptions are correct. First it should be remembered that the surroundings in which the pressure is isotropic are not able to do work on the sample. This means that the energy entering the sample through the free surface must in some way be delivered to it by the upper and/or the lower disk. In the Appendix the forces that should be delivered by the disks to the free surface in order that the stresses at the free surface are as given by [11] are calculated. Again, internally the picture appears to be consistent.

Remains, however, the question whether a free surface may indeed be supposed to be able to produce the stresses that are required to maintain the local force equilibrium between the sample and its surroundings. A mechanism which can produce a certain type of stress is interfacial tension. If the surface is curved, at proper values of the interfacial tension and of the local curvatures of the surface a stress parallel to the local normal on the surface may be compensated. Stresses which are not parallel to the surface normal, however, cannot be compensated by the surface tension mechanism.

From the eqs. [11] and [7] we see that in our case $\boldsymbol{t}_{f}$ and $\boldsymbol{n}_{f}$ are not parallel to each other. Stronger even, it is impossible to find a smoothly curved shape of the free surface such that $t_{f}$ and $\boldsymbol{n}_{f}$ are parallel in every point of it. The reason for this is that the stress tensor $T$, given by [8], does not depend on place and has, in general, non-equal principal values. The vectors $\boldsymbol{t}$ and $\boldsymbol{n}$ are therefore only parallel to each other along three sets of mutually perpendicular flat planes. A similar statement can be made for the ERD-flow with a superimposed torsional flow, which has been proposed in the references (1) and (2).

So we must decide that, whether the flow field in the bulk is as assumed primarily or modified in the way mentioned above, a mechanism which is not provided by surface tension alone is required to take care of the discontinuity of the stress in the plane formed by the free surface. Such a mechanism might be provided by a deviation of the flow field in a relatively thin layer of the material along the free surface from the flow field in the bulk of the sample. These deviations should be such that - in agreement with the constitutive equation of the material - the stress changes from purely isotropic at the free surface itself to a stress in the bulk as given by [11]. In that case the upper disk still does not do work on that part of the upper boundary of the material in which the supposed bulk flow prevails. But it does do work on the narrow outer ring of the sample in which the flow deviates from this. This picture is still consistent with our statement that energy can enter into the (bulk of the) sample through that part of its surface which is not in contact with the plates.

In the foregoing we have emphasized the role of the free surface in the force and energy balances. One of our conclusions was that a superposition of a torsional flow on the primarily assumed bulk flow (3) could, alone, not provide a mechanism to take care of the stress discontinuity at the free surface. It is not unlikely, however, that in reality both 'deviations' - a superimposed torsional flow in the bulk and deviations of the velocity field in the free surface layer - are present at the same time. As a matter of fact, experiments by Payvar and Tanner (1) have demonstrated a velocity lag between the upper and lower disk, with a magnitude in accordance with the value calculated on the basis of an assumed superimposed torsional flow, does exist. Their experiments, however, were carried out with a 'cup-like' lower disk. So in this case there is no free surface of the kind considered in the present paper. Similar experiments reported by Davis and Macosco (2), however, were carried out with a regular flat lower disk and there, too, a velocity lag between the disks of about the predicted magnitude is shown. But in this case there seems to remain a 
small but systematic difference between the measured and the predicted values of the velocity lag. We are inclined to attribute this difference to the stresses in the free surface in the sense discussed in the present paper.

\section{Appendix}

In this appendix we investigate some aspects of the stress balance in the free surface. Although - as mentioned before - in reality there will be some kind of a free surface layer, the problem will be treated here as two-dimensional. As a consequence of this. besides surface stress densities, line stress densities along the boundaries of a surface element will enter into the force balances.

We consider a ring-shaped element of the surface bounded by two circles parallel to the plates located at $z$ and $z+d z$ respectively. The $z$-component of the torque with respect to the center of the lower plate resulting from the traction $\boldsymbol{t}_{f}$ on the ring becomes (cf. eq. [16]):

$$
d M_{f}\langle z\rangle=\pi R^{2} \psi^{2} \omega \eta^{\prime} d z .
$$

Since no external stresses are acting directly on the free surface, this torque must be compensated by the one resulting from the line force densities in the circle which form the boundaries of the ring. If the $z$-component of this torque in a circle at a height $z$ amounts $S(z)$ the equilibrium of torques in the ring requires that

$d M_{f}\langle z\rangle-\left(S+\frac{d S}{d z} d z\right)+S=0$.

So by [A 1] the differential equation for $S(z)$ becomes: $\frac{d S}{d z}=\pi R^{2} \psi^{2} \omega \eta^{\prime}$

Since not net torque is exerted to the lower disk, we have the following boundary condition for $S(z)$ : $S(0)=0$.

So the solution of [A 3] becomes:

$S(z)=\pi R^{2} \psi^{2} \omega \eta^{\prime} z$.

For $z=h$ this is the couple which is delivered by the edges of the upper plate to the free surface; it is also the couple by which the couple [14] acting upon the upper surface is compensated.

The rate of energy input into the free surface by the edge of the upper plate amounts

$W=S(h) \omega=\pi R^{2} \psi^{2} h \omega^{2} \eta^{\prime}$, which by [18] just equals the rate of energy dissipation in the fluid.

\section{Summary}

The balances of torque and energy in the flow between eccentric rotating dises (ERD) have been investigated. Special attention has been given to the role of stresses resulting from the forces that are delivered to the free surface by the edges of the plates. These stresses are shown to be significant in considering the so-called stress power paradox. Their role with respect to deviations of the velocity profile from the usually assumed one is discussed.

\section{Zusammenfassung}

Die Drehmoment- und Energiebilanzen werden für die Strömung zwischen zwei exzentrisch rotierenden Kreisscheiben (ERD) untersucht. Besondere Aufmerksamkeit verdient dabei die Rolle der Spannungen, die die Ränder der Platten auf die freie Oberfläche ausüben. Es wird gezeigt, daß die Anwesenheit dieser Spannungen wesentlich für die Beurteilung des sogenannten Spannungsenergie-Paradoxes ist. Auch wird der $\mathrm{Zu}$ sammenhang zwischen diesen Spannungen und den Abweichungen der wirklichen Geschwindigkeitsverteilung von den bisher angenommenen Verteilungen diskutiert.

\section{References}

1) Paycar, P., R. I. Tanner, Trans. Soc. Rheol. 17. 449 (1973)

2) Daris. W. M., C.W. Macosco. AIChE J. 20. 600 (1974)

3) Blyer, Jr., L. L., S. J. Kurtz, J. Appl. Polymer Sci. 11. 127 (1967).

4) Hutton, J. F.. In: Theoretical Rheology, p. 365 (London 1975).

5) Macosco, C. W., W. M. Davis, Rheol. Acta 13, 814 (1974).

6) Bird. R. B., E. K. Harris. Jr.. AIChE J. 14, $758(1968)$.

7) Abbott, T. N. G., K. Walters, J. Fluid Mech. 40, $205(1970)$.

8) Waterman, H. A., Rheol. Acta 15, 444 (1976).

Authors' address:

Drs. R. J. J. Jongschaap,

Prof. Dr. P.F. Mijnlieff

Department of Applied Physics

Twente University of Technology

Enschede (The Netherlands) 